\title{
EPR studies of the triangular-lattice antiferromagnet $\mathrm{Cs}_{2} \mathrm{CuBr}_{4}$
}

\author{
E. Schulze ${ }^{1,2}$, A.N. Ponomaryov ${ }^{1}$, J. Wosnitza ${ }^{1,2}$, H. Tanaka ${ }^{3}$, and S.A. Zvyagin ${ }^{1}$ \\ ${ }^{1}$ Dresden High Magnetic Field Laboratory (HLD-EMFL) \\ Helmholtz-Zentrum Dresden-Rossendorf, D-01328 Dresden, Germany \\ E-mail: s.zvyagin@hzdr.de \\ ${ }^{2}$ Institut für Festkörperphysik, TU Dresden, D-01069 Dresden, Germany \\ ${ }^{3}$ Tokyo Institute of Technology, Tokyo 152-8550, Japan
}

Received June 19, 2017, published online September 25, 2017

\begin{abstract}
The spin dynamics of the spin-1/2 triangular-lattice antiferromagnet $\mathrm{Cs}_{2} \mathrm{CuBr}_{4}$ is probed by means of highfrequency electron paramagnetic resonance (EPR) spectroscopy. Temperature dependences of EPR parameters are studied in a broad temperature range between 1.4 and $200 \mathrm{~K}$ for different orientations of the applied magnetic field. In the high-temperature regime $\left(T>>J / k_{B}\right)$, an unusually broad and anisotropic resonance line is detected, suggesting a sizeable Dzyaloshinskii-Moriya interaction. Employing the theory of exchange narrowing, the ratio of the Dzyaloshinskii-Moriya vector components, $D_{C} / D_{a} \approx 0.3$, is estimated.

PACS: 75.50.Ee Antiferromagnetics;

76.30.-v Electron paramagnetic resonance and relaxation;

76.50.+g Ferromagnetic, antiferromagnetic, and ferrimagnetic resonances; spin-wave resonance.
\end{abstract}

Keywords: antiferromagnet, EPR, Dzyaloshinskii-Moriya interaction.

A spin-1/2 Heisenberg antiferromagnet (AF) on a triangular lattice is one of the best model systems to probe effects of quantum fluctuation and frustrations in strongly correlated spin systems, exhibiting unusual ground-state properties and a rich excitation spectrum. It was suggested, that quantum fluctuations can be strong enough to destroy the classical $120^{\circ}$ order in triangular-lattice spin-1/2 Heisenberg AFs [1], resulting in a two-dimensional (2D) spin liquid with the excitation spectrum formed by fractionalized quasiparticles, spinons. Although the existence of such a 2D spin liquid has been predicted more than four decades ago, theoretical identification and experimental detection of this state of matter has proved challenging.

In general, the Hamiltonian of a spin-1/2 systems with a triangular lattice in zero field is given by

$$
\mathcal{H}=J \sum_{\langle i, j\rangle} \mathbf{S}_{i} \cdot \mathbf{S}_{j}+J^{\prime} \sum_{\left\langle i, j^{\prime}\right\rangle} \mathbf{S}_{i} \cdot \mathbf{S}_{j^{\prime}}+\mathcal{H}_{\delta},
$$

where $\mathbf{S}_{i}, \mathbf{S}_{j}$, and $\mathbf{S}_{j^{\prime}}$ are spin-1/2 operators at sites $i, j$, and $j^{\prime}$, respectively, $J$ and $J^{\prime}$ are the exchange-coupling parameters along the horizontal and zigzag bonds [Fig. 1(a)], respectively, and $\mathcal{H}_{\delta}$ represents various possible small anisotropic contributions. Depending on the $J^{\prime} / J$ ratio, theory [2-7] predicts a rich variety of magnetic struc- tures, ranging from the $1 \mathrm{D}$ collinear phase to an incommensurate triangular order.

The spin-1/2 triangular-lattice $\mathrm{AFs} \mathrm{Cs}_{2} \mathrm{CuCl}_{4}$ and $\mathrm{Cs}_{2} \mathrm{CuBr}_{4}$ are among the most intensively studied representatives of this class of low-D frustrated materials. These two materials have many common properties. Both have a slightly distorted layered arrangement of $\mathrm{Cu}^{2+}$ ions in a triangular pattern [Fig. 1(b)]. The materials are character- (a)

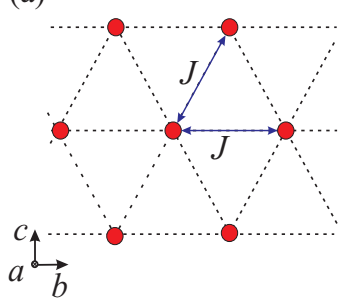

(b)

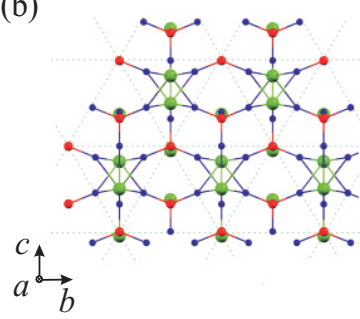

Fig. 1. (Color online) Schematic picture of exchange paths in $\mathrm{Cs}_{2} \mathrm{CuBr}_{4}$ in the $b c$ plane (a). Schematic view of the crystal structure of $\mathrm{Cs}_{2} \mathrm{CuBr}_{4}$ in the $b c$ plane. The $\mathrm{Cu}^{2+}$ ions form the triangular lattice. Red circles represent copper ions, while green and blue circles represent cesium and bromine ions, respectively (b). 
ized by the same scale of in-plane spin interactions, $J / k_{B}=4.7 \mathrm{~K}, J^{\prime} / J \simeq 0.3$ and $J / k_{B}=14.9 \mathrm{~K}, J^{\prime} / J \simeq 0.41$ for $\mathrm{Cs}_{2} \mathrm{CuCl}_{4}$ and $\mathrm{Cs}_{2} \mathrm{CuBr}_{4}$, respectively [8,9]. Due to finite interplane interactions, $\mathrm{Cs}_{2} \mathrm{CuCl}_{4}$ and $\mathrm{Cs}_{2} \mathrm{CuBr}_{4}$ undergo transitions into magnetically ordered incommensurate states, at $T_{N}=0.62$ and $1.4 \mathrm{~K}$, respectively [10,11]. In spite of these (and many other) similarities, the magnetic properties of $\mathrm{Cs}_{2} \mathrm{CuBr}_{4}$ appear much richer, including $1 / 3$ and 2/3 saturation magnetization plateaus [12] and a number of additional (still unidentified) field-induced phase transitions [13]. Most importantly, the observation of a relatively large zero-field energy gap, $\Delta \sim 0.7 \mathrm{~J}$, in the excitation spectrum of $\mathrm{Cs}_{2} \mathrm{CuBr}_{4}[9,14]$ appears to be inconsistent with the $1 \mathrm{D}$ scenario, proposed for $\mathrm{Cs}_{2} \mathrm{CuCl}_{4}$ $[15,16]$. One of the critical issues is to clarify the reason for such a big difference of the magnetic properties of these two frustrated materials.

Electron paramagnetic resonance (EPR, also known as electron spin resonance, ESR) spectroscopy is recognized as a very powerful tool to probe the anisotropy of magnetic interactions in strongly correlated spin systems. Recently performed EPR studies of $\mathrm{Cs}_{2} \mathrm{CuCl}_{4}$ [17] provided the important information on the magnetic anisotropy in this material. It was shown that the main source of the hightemperature EPR line broadening is the DzyaloshinskiiMoriya (DM) interaction. Employing a high-temperature approximation, the vector components of the DM interaction, $D_{a}=0.33 \mathrm{~K}$ and $D_{c}=0.36 \mathrm{~K}$, were determined from the EPR angular dependence in the $a c$ plane.

$\mathrm{Cs}_{2} \mathrm{CuBr}_{4}$ realizes a distorted triangular lattice with orthorhombic crystal structure (space group Pnma) and the room-temperature lattice parameters $a=10.195 \AA$, $b=7.965 \AA, c=12.936 \AA$, and $Z=4$ (the number of formula units per unit cell) [18]. High-quality single crystals of $\mathrm{Cs}_{2} \mathrm{CuBr}_{4}$ were grown by slow evaporation of aqueous solutions of $\mathrm{CsBr}$ and $\mathrm{CuBr}_{2}$ similar as described in [18]. Due to a relatively broad line, no resonance absorption was detected using the standard $X$-band EPR spectrometer. The EPR measurements were performed employing a high-frequency transmission-type spectrometer equipped with a $16 \mathrm{~T}$ superconducting magnet, similar to that described in Ref. 19. A VDI modular transmitter (product of Virginia Diodes Inc., USA) was used as a mm-wave radiation source. The EPR measurements were performed at temperatures down to $1.4 \mathrm{~K}$.

The temperature dependences of the EPR linewidth and effective $g$ factors measured at a frequency of 312 $\mathrm{GHz}$ for three field orientations are shown in Figs. 2 and 3, respectively. One can see that the linewidth and $g$ factor do not show any significant temperature dependence down to approximately 40-50 K. Below this temperature, the EPR field shifts and line broadening is observed. Such a behavior is an indication of the combined effect of exchange and anisotropic spin-spin interactions. At approximately $14 \mathrm{~K}$, the EPR line splits (the splitting was

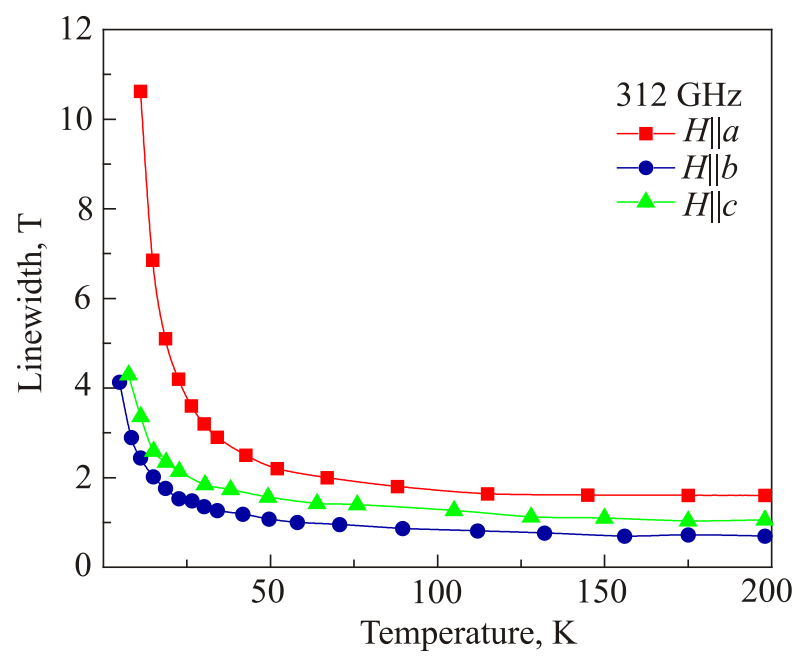

Fig. 2. (Color online) Temperature dependence of the EPR linewidths measured at $312 \mathrm{GHz}$. The data for $H \| a, b$ and $c$ are labeled by squares, circles, and triangles, respectively. The lines are guides for the eye.

observed for $H \| a, c$; some selected spectra for $H \| a$ are shown in Fig. 4), eventually opening a gap, $\Delta \approx 9.5 \mathrm{~K}$, in the excitation spectrum $[9,14]$. From comparison to results of harmonic spin-wave theory calculations for the spin-1/2 triangular AF model (with parameters as defined in Ref. 9) the observed gapped mode was identified as a magnetic excitation at the Brillouin zone boundary, which is observable in $\mathrm{Cs}_{2} \mathrm{CuBr}_{4}$ due to the staggered DM interaction [14].

Above approximately $50 \mathrm{~K}$, the system is in the hightemperature regime $\left(T>>J / k_{B}\right)$. Remarkably, the EPR linewidth is almost constant at least up to $200 \mathrm{~K}$, suggesting that the phonon-assisted spin-lattice broadening (which should be proportional to the temperature) is not relevant.

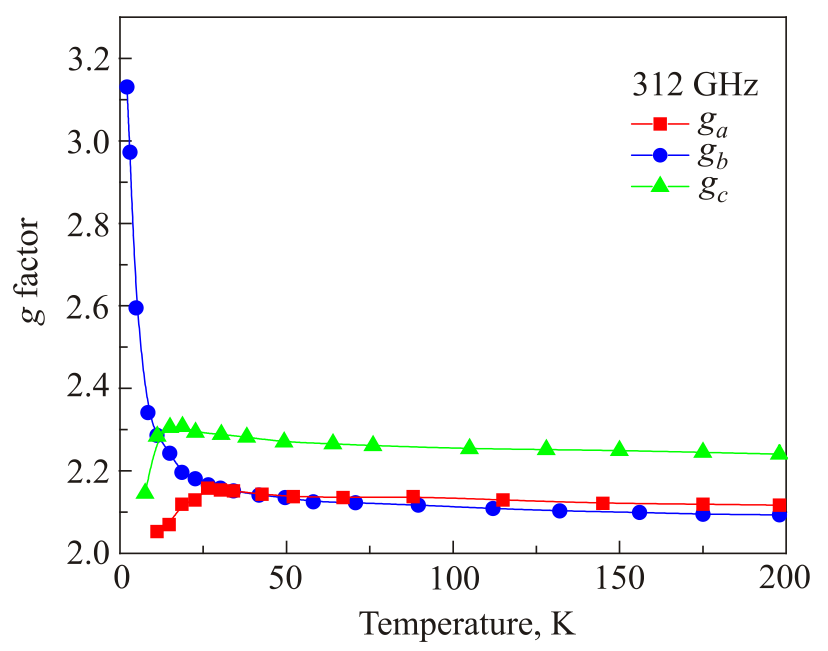

Fig. 3. (Color online) Temperature dependence of the effective $g$ factor measured at $312 \mathrm{GHz}$. The data for $H \| a, b$ and $c$ are labeled by squares, circles, and triangles, respectively. Note that data for $H \| a, c$ are shown only down to $14 \mathrm{~K}$ (below this temperature the EPR lines split). The lines are guides for the eye. 


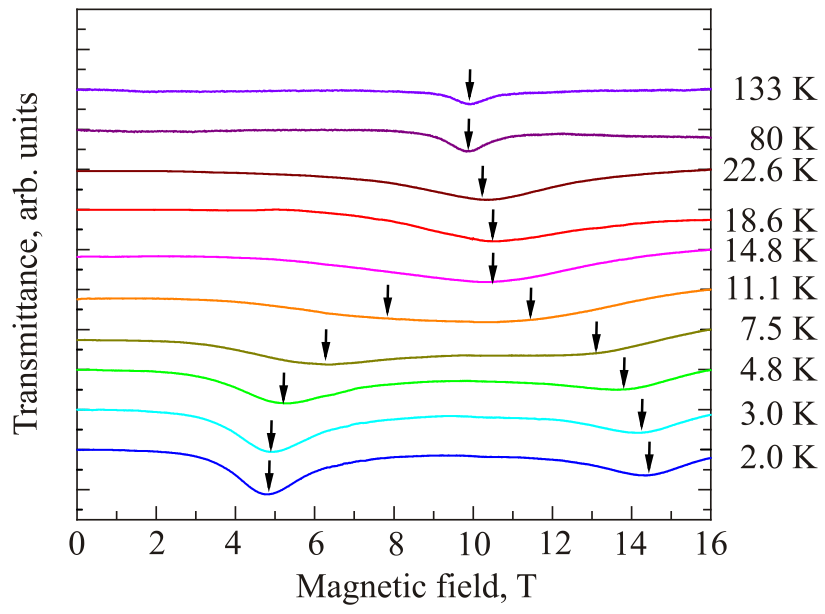

Fig. 4. (Color online) Selected EPR spectra measured at $312 \mathrm{GHz}$ at different temperatures, $H \| a$. Resonance absorption maxima are denoted by arrows.

Three spectra obtained at a frequency of $285 \mathrm{GHz}$ for different field orientations ( $T=135 \mathrm{~K})$ are shown in Fig. 5. The spectra were fit using the Lorentzian line shape, revealing $g_{a}=2.17(2), g_{b}=2.10(2), g_{c}=2.22(2)$ and EPR full widths at half maximum (FWHM) $\Delta H_{a}=1.75(1) \mathrm{T}$, $\Delta H_{b}=0.60(1) \mathrm{T}, \Delta H_{c}=1.00$ (3) $\mathrm{T}$ for $H \| a, b$, and $c$, respectively. It is important to mention, that contrary to many other spin-1/2 exchange-coupled spin systems with spin interactions of the same order of magnitude [20-23], the high-temperature EPR line in $\mathrm{Cs}_{2} \mathrm{CuBr}_{4}$ is up to two orders of magnitude broader (for comparison, the EPR linewidth in the isostructural compound $\mathrm{Cs}_{2} \mathrm{CuCl}_{4}$ measured at $T=100 \mathrm{~K}$ is $\sim 0.02-0.03 \mathrm{~T}$ [17], which is about 50 times smaller than the EPR linewidth in $\mathrm{Cs}_{2} \mathrm{CuBr}_{4}$ ). Let us discuss possible contributions, which affect the EPR linewidth.

(i) Following the conventional estimates [24] and assuming that the distance between neighboring $\mathrm{Cu}^{2+}$ ions is $\sim 8 \AA$, the contribution to the dipole-dipole interaction appears to be negligibly small ( $\sim 1$ Oe).

(ii) The anisotropic Zeeman contribution can be calculated using the equation [25]

$$
\Delta H_{A Z}=\left(\frac{\Delta g}{g}\right)^{2} \frac{g \mu_{B} H_{\mathrm{res}}^{2}}{\sqrt{\left\langle J^{2}\right\rangle}},
$$

where $\Delta g=g_{c}-g_{b}, g=g_{a, c}$, and $\left\langle J^{2}\right\rangle=\frac{1}{3}\left(J^{2}+2 J^{\prime 2}\right)$ or $\langle J\rangle \approx 10 \mathrm{~K}$. As result, one obtains $\Delta H_{A Z} \approx 0.04 \mathrm{~T}$, which is much smaller than the measured EPR linewidth.

(iii) Rough estimates of two remaining major contributions to the EPR linewidth, the antisymmetric DM interaction and symmetric anisotropic interaction, give $D \propto(\Delta g / g)\langle J\rangle=1.65 \mathrm{~K}$ and $E \propto(\Delta g / g)^{2}\langle J\rangle=0.2 \mathrm{~K}$, respectively [26]. Based on that, the DM interaction can be regarded as the main reason of the broad anisotropic EPR line observed in our experiments.

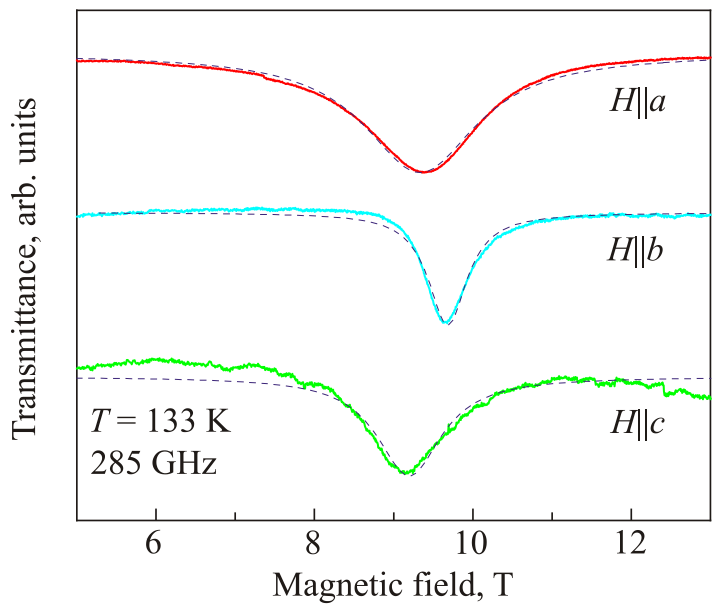

Fig. 5. (Color online) EPR spectra measured at $285 \mathrm{GHz}, T=135 \mathrm{~K}$, with magnetic field applied along the three different crystallographic axes. The dashed lines are Lorentzian fit results.

Figure 6 shows the angular dependence of the EPR linewidth with magnetic field applied in the ac plane, obtained at $312 \mathrm{GHz}$ and $T=80 \mathrm{~K}$.

If the EPR line is of Lorentzian shape, the FWHM $\Delta H$ can be calculated employing the theory of exchange narrowing [27] as

$$
\Delta H=2 C\left(\frac{M_{2}^{3}}{M_{4}}\right)^{1 / 2},
$$

where $M_{2}$ and $M_{4}$ are the second and fourth moment, $C$ is a dimensionless constant, $C=\pi / \sqrt{2}$ [17]. Following the approach from Ref. 17, 27, the FWHM can be written as

$$
\Delta H=C \frac{D^{2}(\alpha, \beta) k_{B}}{\langle J\rangle g \mu_{B}} \frac{1}{\sqrt{2}} .
$$

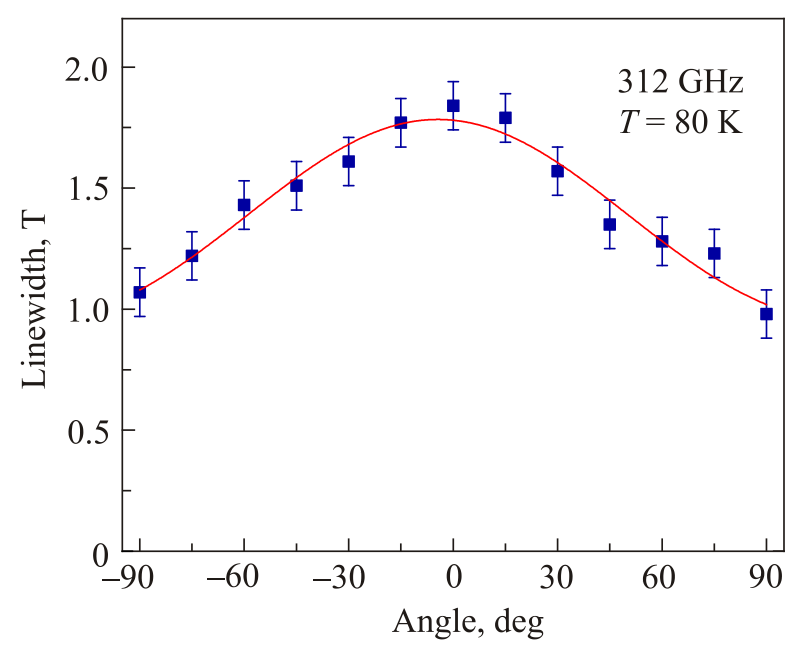

Fig. 6. (Color online) Angular dependence of the EPR linewidth with magnetic field applied in the ac plane, obtained at $312 \mathrm{GHz}$, $T=80 \mathrm{~K} .0^{\circ}$ corresponds to $H \| a$, while $\pm 90^{\circ}$ correspond to $H \| c$. The line is a guide for the eye. 
Here, $k_{B}$ is the Boltzmann constant, $\mu_{B}$ is the Bohr magneton, and

$$
\begin{aligned}
\mathcal{D}^{2}(\alpha, \beta) & =D_{a}^{2}\left(1+\sin ^{2} \beta \cos ^{2} \alpha\right)+D_{c}^{2}\left(1+\cos ^{2} \beta\right)+ \\
& +D_{a} D_{c} \cos \alpha \sin 2 \beta,
\end{aligned}
$$

where $D_{a}$ and $D_{c}$ are the DM vector components along the $a$ and $c$ axes, respectively. The angles $\alpha$ and $\beta$ are defined by

$$
\begin{gathered}
\cos \alpha=\frac{A}{\sqrt{A^{2}+B^{2}}}, \quad \cos \beta=\frac{C}{\sqrt{A^{2}+B^{2}+C^{2}}}, \\
A=g_{a a} \sin \theta \cos \varphi+g_{a b} \sin \theta \sin \varphi+g_{a c} \cos \theta, \\
B=g_{c a} \sin \theta \cos \varphi+g_{c b} \sin \theta \sin \varphi+g_{c c} \cos \theta . \\
C=g_{b a} \sin \theta \cos \varphi+g_{b b} \sin \theta \sin \varphi+g_{b c} \cos \theta,
\end{gathered}
$$

Here, $\varphi$ denotes the azimuthal angle counted from the $a$ axis and $\theta$ denotes the polar angle counted from the $c$ axis.

Taking into account that the magnetic field is applied in the $a c$ plane (the DM interaction is not allowed along the $b$ axis), we can neglect contributions to the moments for $\varphi \neq 0$. Our analysis provides $D_{a}=(4.7 \pm 0.5) \mathrm{K}$ and $D_{C}=(1.6 \pm 0.3) \mathrm{K}$ for the DM components along the $a$ and $c$ axes, respectively. These numbers appear to be too big, compared to $\langle J\rangle=10 \mathrm{~K}$, questioning the validity of the used approach for compounds with a substantial DM interaction. Nevertheless, the approach can be employed for estimation of the $D_{c} / D_{a}$ ratio. In our calculations, we neglect offdiagonal elements of the $g$ tensor; the corresponding error due to each off-diagonal element does not exceed $\sim 10 \%$. Based on these considerations, the ratio $D_{c} / D_{a}$ can be written as

$$
\frac{\Delta H_{C}}{\Delta H_{a}}=\left(\frac{M_{2}^{3}\left(\varphi=0^{\circ}, \theta=0^{\circ}\right) / M_{4}\left(\varphi=0^{\circ}, \theta=0^{\circ}\right)}{M_{2}^{3}\left(\varphi=0^{\circ}, \theta=90^{\circ}\right) / M_{4}\left(\varphi=0^{\circ}, \theta=90^{\circ}\right)}\right)^{1 / 2} .
$$

Using the function $\mathcal{D}^{2}$ as introduced in (5) and keeping $\varphi=0^{\circ}$ fixed leads to

$$
\begin{aligned}
\left(\frac{M_{2}^{3}\left(\theta=0^{\circ}\right) / M_{4}\left(\theta=0^{\circ}\right)}{M_{2}^{3}\left(\theta=90^{\circ}\right) / M_{4}\left(\theta=90^{\circ}\right)}\right) & =\left(\frac{\mathcal{D}^{2}\left(\theta=0^{\circ}\right)}{\mathcal{D}^{2}\left(\theta=90^{\circ}\right)}\right)^{2}= \\
& =\left(\frac{2 D_{c}^{2}+D_{a}^{2}}{2 D_{a}^{2}+D_{c}^{2}}\right)^{2},
\end{aligned}
$$

we obtain $D_{c} / D_{a}=0.3(1)$.

In conclusion, we performed high-frequency EPR studies of the spin-1/2 triangular-lattice antiferromagnet $\mathrm{Cs}_{2} \mathrm{CuBr}_{4}$. In the high-temperature regime $\left(T>>J / k_{B}\right)$, a very broad and anisotropic resonance line was detected, suggesting the presence of a sizeable DM interaction. Employing the EPR exchange narrowing theory, the ratio of the DM vector components, $D_{c} / D_{a} \approx 0.3$, was estimated. Our studies strongly suggest that the effect of the DM in- teraction is essential and needs to be taken into account, when explaining the cascade of the field-induced transitions and other unusual magnetic properties revealed in $\mathrm{Cs}_{2} \mathrm{CuBr}_{4}$ at low temperatures.

\section{Acknowledgements}

This work was supported by the Deutsche Forschungsgemeinschaft (DFG, Germany) and by the HLD at HZDR, member of the European Magnetic Field Laboratory (EMFL). The authors would like to thank M.S. Henriques for the help orienting samples.

1. P.W. Anderson, Mater. Res. Bull. 8, 153 (1973).

2. O.A. Starykh, H. Katsura, and L. Balents, Phys. Rev. B 82, 014421 (2010).

3. S. Ghamari, C. Kallin, S.S. Lee, and E.S. Sørensen, Phys. Rev. B 84, 174415 (2011).

4. D. Heidarian, S. Sorella, and F. Becca, Phys. Rev. B 80, 012404 (2009).

5. M.Q. Weng, D.N. Sheng, Z.Y. Weng, and R.J. Bursill, Phys. Rev. B 74, 012407 (2006).

6. A. Weichselbaum and S.R. White, Phys. Rev. B 84, 245130 (2011).

7. J. Reuther and R. Thomale, Phys. Rev. B 83, 024402 (2011).

8. R. Coldea, D.A. Tennant, K. Habicht, P. Smeibidl, C. Wolters, and Z. Tylczynski, Phys. Rev. Lett. 88, 137203 (2002).

9. S.A. Zvyagin, D. Kamenskyi, M. Ozerov, J. Wosnitza, M. Ikeda, T. Fujita, M. Hagiwara, A.I. Smirnov, T.A. Soldatov, A.Y. Shapiro, J. Krzystek, R. Hu, H. Ryu, C. Petrovic, and M.E. Zhitomirsky, Phys. Rev. Lett. 112, 077206 (2014).

10. R. Coldea, D.A. Tennant, R.A. Cowley, D.F. McMorrow, B. Dorner, and Z. Tylczynski, J. Phys. Condens. Matter 8, 7473 (1996).

11. T. Ono, H. Tanaka, H. Aruga Katori, F. Ishikawa, H. Mitamura, and T. Goto, Phys. Rev. B 67, 104431 (2003).

12. T. Ono, H. Tanaka, O. Kolomiyets, H. Mitamura, F. Ishikawa, T. Goto, K. Nakajima, A. Oosawa, Y. Koike, K. Kakurai, J. Klenke, P. Smeibidle, M. Meissner, R. Coldea, A.D. Tennant, and J. Ollivier, Theor. Phys. Suppl. 159, 217 (2005).

13. N.A. Fortune, S.T. Hannahs, J. Yoshida, T.E. Sherline, T. Ono, H. Tanaka, and Y. Takano, Phys. Rev. Lett. 102, 257201 (2009).

14. S.A. Zvyagin, M. Ozerov, D. Kamenskyi, J. Wosnitza, B.J. Krzystek, D. Yoshizawa, M. Hagiwara, R. Hu, H. Ryu, C. Petrovic, and M.E. Zhitomirsky, New J. Phys. 17, 113059 (2015).

15. M. Kohno, O.A. Starykh, and L. Balents, Nat. Phys. 3, 790 (2007).

16. K.Yu. Povarov, A.I. Smirnov, O.A. Starykh, S.V. Petrov, and A.Ya. Shipiro, Phys. Rev. Lett. 107, 037204 (2011).

17. M.A. Fayzullin, R.M. Eremin, A. Dittl, N. van Well, F. Ritter, W. Aßmus, J. Deisenhofer, H.-A. Krug von Nidda, and A. Loidl, Phys. Rev. B 88, 174421 (2013). 
18. B. Morosin and E.C. Lingafelter, Acta Crystallogr. 13, 807 (1960).

19. S.A. Zvyagin, J. Krzystek, P.H.M. van Loosdrecht, G. Dhalenne, and A. Revcolevschi, Physica B 346-347, 1 (2004).

20. B. Pilawa, J. Phys.: Condens. Matter 9, 3779 (1997).

21. H.-A. Krug von Nidda, L.E. Svistov, M.V. Eremin, R.M. Eremina, A. Loidl, V. Kataev, A. Validov, A. Prokofiev, and W. Aßmus, Phys. Rev. B 65, 134445 (2002).

22. A.A. Validov, M. Ozerov, J. Wosnitza, S.A. Zvyagin, M.M. Turnbull, C.P. Landee, and G.B. Teitel'baum, J. Phys.: Condens. Matter 26, 026003 (2014).
23. V.N. Glazkov, M. Fayzullin, Yu. Krasnikova, G. Skoblin, D. Schmidiger, S. Mühlbauer, and A. Zheludev, Phys. Rev. B 92, 184403 (2015).

24. I. Yamada, M. Nishi, and J. Akimutsu, J. Phys.: Condens. Matter 8, 2625 (1996).

25. D.L. Huber, G. Alejandro, A. Caneiro, M.T. Causa, F. Prado, M. Tovar, and S.B. Oseroff, Phys. Rev. B 60, 12155 (1999).

26. T. Moriya, Phys. Rev. B 120, 92 (1960).

27. T.G. Castner, Jr. and M.S. Seehra, Phys. Rev. B 4, 38 (1971). 